\title{
A Routing Algorithm to Optimize the Energy Efficacy of Nodes in Internet of Things
}

\author{
Ananya Roy \\ Dept. of Computer Science \\ Assam University, \\ Silchar India
}

\author{
Prodipto Das \\ Dept. of Computer Science \\ Assam University, \\ Silchar India
}

\author{
Rajib Das \\ Dept of Computer Science \\ Karimganj College \\ Assam, India
}

\begin{abstract}
The Internet of Things (IoT) is a powerful example that has made advancement in the almost every arena of human life. Its primary aim is that of connecting even the most mundane objects to provide a comfortable lifestyle. Hence, one of the major factors in IoT is the security issue. In this paper we will try to optimize the energy problems which occur for securing Internet of Things. For example, using the RFID labels, sensors etc, IoT have volunteered out from its previous versions and has changed the internet into a harmonized Future Internet. But with these advancements arises the need for high energy consumption. Our main goal is to propose an energy efficient protocol to improve the energy efficiency of nodes thereby securing the IoT. The authentication of this procedure is established in an IoT atmosphere with separate $\mathrm{C}$ platform.
\end{abstract}

\section{Keywords}

IoT, RFID, sensors, protocol, energy efficiency.

\section{INTRODUCTION}

In today's world of internet, everything object can be connected with each other. But, in terms of network connectivity, all devices cannot be connected with the network, which limit the level of task. To make this task unlimited, and perform all the task in a smooth manner one to one connection is required between all the devices. But for this the present day internet is one of the constraint, which disrupts the connectivity. But IoT has emerged as one of the technology, which has enabled every individual object to communicate among themselves [1-3].In the process of communication, information will spawn and we should ensure access control. IoT can be used in various fields of our daily life like health, transportation etc. Various sensors networks, RFID are the main ingredients for communication between object to object in IoT. For the Internet of Things to emerge successfully, it needs to go beyond the traditional computing and allow our daily objects to be connected with the environment.

In the IoT network, the nodes distributed in various parts of the network collect the information required by them and transfer among themselves [4-6]. For the transfer of information between the nodes, inadvertent energy is required by the starting node for forwarding the data packet which results in network partitioning inducing very high energy depletion. Thus, the network performance is basically exaggerated by the energy depletion [7]. To find the straight route between one hop to another, hop count is used by most of the routing protocols. However, in the millions of devices connected to the internet, the communication architecture and the rate of data transfer cannot stand the energy required by the IoT devices.

\section{PROBLEM IDENTIFICATION AND SIGNIFICANCE}

In the IoT network, where only one or two devices are required for making the whole communication network, the nodes can be connected to each other very easily [8]. But if we want to deploy IoT network where we can ensure communication between the people and devices as well as with the devices themselves, then arise the need for a complex network and also this type of network needs to spread to all regions for definite tasks. Conversely, installing IoT devices universally with billions of consumers is a giant task [9].

With the advent of Internet of Things, various applications will be compressed. However, various factors like scale, network availability, consumer participation, exposure etc. will affect each type of applications. The applications can be categorized as follows: (i) Home (ii) Personal (iii) Healthcare (iv) Mobile.

For ensuring to give the best network for communication among the devices, we come through various challenges for connectivity, the supreme and noteworthy concern being the system energy intake.

Proper routing is required for the nodes to forward data collected from the various sensors to intermediary node. In the whole process of data transmission from the starting node to the target node, the nodes use vast quantity of energy which outcomes in energy waste and hints to network splitting. Therefore energy effectiveness is a vital concern which affects the whole network performance in the whole IoT network [10].

To allow the devices to transmit data effortlessly between them and the connected servers and other system we need an energy efficient routing in the network.

\section{LITERATURE REVIEW}

In this part, the features of the existing protocols are discussed. Iot is very successfully in gaining peoples attraction because of its vide variety of features. With this advent the data transfer rate has also increased day by day. For all this the network should have to be capable for the vast quantity of records transfer as because continuous use of clever devices hints to amplified data movement which the network can handle which results in service degradation.Spirent's diameter testing application estimates the network node by node and thus helps to relate to the realworld conditions. Also this can be used to evaluate the working of new devices on the existing network. [11].The Ad- 
hoc routing gives good outcome by taking into consideration the neighbor nodes load capacity to transfer packets [12]. The network performance is improved by data aggregation. It is done by calculating the entropies of data from source node and consolidating the data [13]. The network efficiency is increased by self-localization of the progressing node with its neighboring node. The data history of the neighboring node is checked by forwarding node. And if the data is already available with the previous node than data packet forwarding is avoided by the forwarding node to reduce redundancy [14]. In IoT, records transmission from home to target is a main fear in changing environment. Routing protocols for IoT are classified as table-driven and on-demand driven. In the former, in the routing table the information from neighbouring nodes is constantly maintained. Examples of this category are Destination-Sequenced Distance Vector (DSDV) and Fisheye State Routing (FSR). Whereas, in the on-demand driven, the starting node firsts sends a RREQ packet to the neighbour and then advanced to the neighbouring nodes to rise the area until the path is found. Upon discovery of the route, a reply is directed back via the similar route. Example of on-demand driven protocol are: a) Dynamic source routing(DSR) b)AdHoc on-Demand Distance Vector(AODV) are example of ondemand driven protocol [15].

\section{ENERGY EFFICIENCY OF NODE}

In the offered procedure, first the network controller calculates the average residual energy, for the entire nodes in whole network. To find the average of the residual energy, two aspects are taken into consideration: (1) the middling rate of the residual energy premeditated from the data received from each node by the network controller is known to each node. (2) When all nodes announce the records packets, allowing new nodes know about its existence and as one of the path from the starting to the target, its neighboring node gets to know its residual energy.

With this consideration, we forward the packets from the source to the destination using the least quantity of nodes. Also, only a limited energy is used by every node, with this approach we can ensure low energy consumption. To establish a path for transmission of data packets, the starting node newscasts the RREQ packet. The neighbor node on receipt of the RREQ packet, using the value of the residual energy computes the forwarding probability plus number of transmission required in the proposed algorithm.

Conversely, the node matches the preset residual energy threshold with the average residual energy of every node. If the threshold energy in the network is less than the average rate of residual energy from the entire nodes in the network, then it is assumed by the node that the network is now in a upright form in respect of energy. Thus the forwarding probability is not required to be made higher. The node can thus compute forwarding probability. Conversely, if the reverse happens, that is the average energy is less than the the threshold energy, than it is concluded that the network is not in a good condition and accordingly, the value of forwarding probability is increased by our projected procedure. Figure 1 depicts the data flow of our approach. The detail procedure is as given below:

\subsection{ALGORITHM}

Step 1: Initialise all the values like $M_{t c}, E T_{j-1}, \Delta M_{t c}, \delta, \theta, \sigma$ $, \varepsilon, \mathrm{F}, \mathrm{TH}, \mathrm{LP}$.

Step 2: Input the values: $M_{t c}, E T_{j-1}, \Delta M_{t c}, \delta, \theta, \sigma, \varepsilon, I_{k}$.

Step 3: Calculate FP

Step 4: The Low Hop Count (LC) is set

Step 5: Check if the energy of node $\mathrm{k}$ is greater than the threshold energy, if

$$
\sigma>T H
$$

Step 6: Then set $\varepsilon$, the other node

$$
\begin{aligned}
& \text { Else } \\
& \text { Calculate F }
\end{aligned}
$$

Step 7: If F> SD // we take a decision about the packet if the forwarding probability is greater than the data sent

Else

Discard the data packet

Step 8: End if

Step 9: End

In step 1 all the values are initialized, in the next step (Step 2 \& 3), we take the input and calculate the output. In step 4 , based on the packets arrival, they are forwarded to the next node, which is decide based on the the value of LC. In step 5, we check whether the average energy is higher than the threshold energy. If $\sigma>T H$, then we choose a new node for forwarding the packet or we calculate the F(step 6). In Step 7, we check if the forwarding probability is greater than the data sent and based on the decision, we chose or discard the packets. In Table 1, we find the explanation of the symbols used.

$$
\mathrm{F}=\left[\delta+I_{k}\left[1+\frac{\left(E T_{j-1}, j-\Delta M_{t c}\right)}{1-\Delta M_{t c}}\right]\right]^{1 / \theta}
$$

$$
I_{k}=\left\{\begin{array}{l}
\frac{1-\delta}{2 \times \sigma}, \text { if } \alpha>x \\
\frac{1-\delta}{2 \times \varepsilon}, \text { if } \alpha \leq x
\end{array}\right.
$$


Table 1: Explanation of used symbols

\begin{tabular}{|c|l|}
\hline Symbols & \multicolumn{1}{c|}{ Description } \\
\hline$M_{t c}$ & $\begin{array}{l}\text { Estimated transmission } \\
\text { Amount }\end{array}$ \\
\hline $\mathrm{F}$ & Forwarding Probability \\
\hline$E T_{j-1}$ & $\begin{array}{l}\text { Expected transmission } \\
\text { Amount amongst nodes j-1 } \\
\text { and k }\end{array}$ \\
\hline$\Delta M_{t c}$ & $\begin{array}{l}\text { Maximum Estimated } \\
\text { transmission Amount }\end{array}$ \\
\hline$I_{k}$ & $\begin{array}{l}\text { Original energy of } \mathrm{k}^{\text {th }} \\
\text { node }\end{array}$ \\
\hline$\delta$ & $\begin{array}{l}\text { Previously defined lowest } \\
\text { forwarding probability }\end{array}$ \\
\hline$\theta$ & $\begin{array}{l}\text { Bigger factor for difference of } \\
\text { forwarding probability }\end{array}$ \\
\hline$\sigma$ & $\begin{array}{l}\text { Maximum energy of the } \\
\text { preceding node }\end{array}$ \\
\hline$\varepsilon$ & $\begin{array}{l}\text { Maximum energy of new } \\
\text { node }\end{array}$ \\
\hline TH & Threshold energy \\
\hline$\alpha$ & Average energy of nodes \\
\hline LC & Low hop count \\
\hline & \\
\hline
\end{tabular}

\subsection{Flowchart}

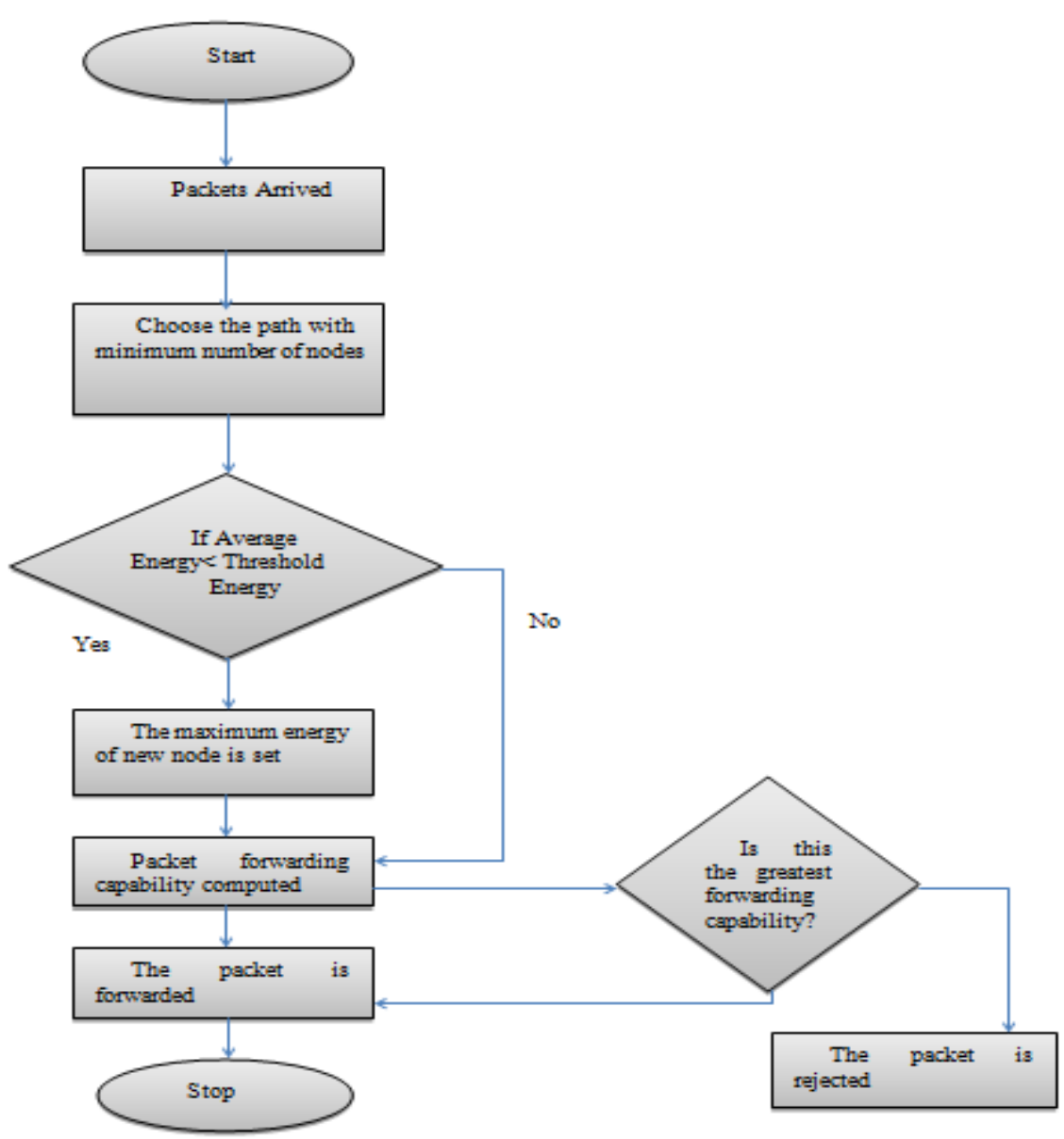

Figure 1: Working process of EEN algorithm

\section{EXPERIMENTAL RESULTS}

For our work we have used $\mathrm{C}$ language and arduino UN-R3 board. The size of our data packet is $1 \mathrm{~KB}$. In this process we have used 360 nodes. The key point of this approach is to quantity the presentation of our algorithm EEN. The setup comprises of 360 nodes. Figure 2 shows our approach. 


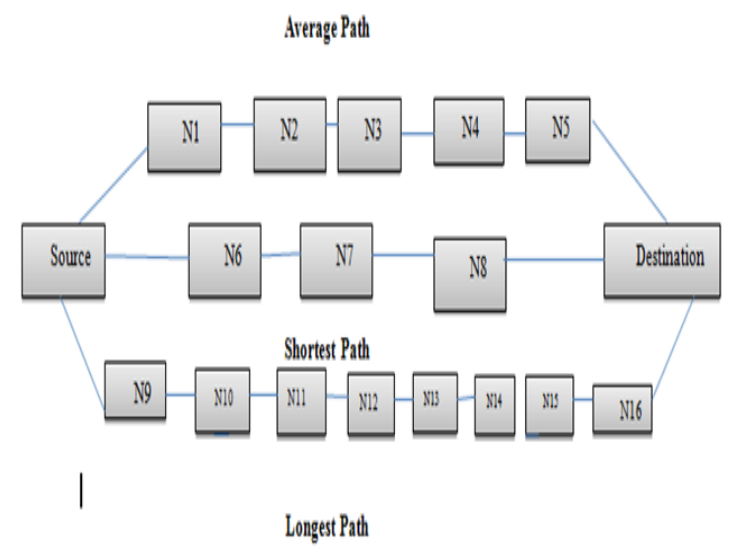

Figure 2: Proposed Algorithm (EEN)

One of the node is the source node and the other is the destination node (Energy of 10 joules) is initialized for each node and a total of $50 \mathrm{~KB}$ data is transferred from the starting to the target node during one turn. Here, there is a total of three different routes for the transmission of packets viz: shortest consisting of 3 nodes, average path contains 5 nodes and the lengthiest route contains 8 nodes. In the shortest path 1 joule of energy is consumed by each node to forward data packets to the next node in the path. In the middling route 2 joule of energy is consumed by each node to forward data packets to its next node in the path. And in the longest path 4 joule of energy is consumed by every node to forward data packets to its next node in the path. 2 joules of energy per node is the threshold energy for this transmission process that means if the nodes residual energy is less than 2 joule then this node cannot be used. Based on this we consider two matrices.

- Energy Efficiency of Node

- $\quad$ Remaining energy of node

\subsection{Energy Efficiency of Node}

From Figure 3, we see the results of our proposed algorithm (EEN) generated by using $C$ language. From figure 4 , we see that until the threshold energy is reached by the nodes, the direct that is the shortest route is chosen on a regular basis. On attainment of threshold energy in the shortest path by the nodes, the middling route is chosen in which the nodes have the supreme energy and also the next straight route to transmit the data packets. Again, from the average path, the nodes will be removed and communication pauses since the energy of all the nodes attain the threshold energy. Table 2 shows the energy consumption (rounded values) of proposed EEN algorithm and Figure 3 shows the graph to find the routes. Table 3 shows the nodes residual energy using EEN along with other protocols.

\subsection{Remaining Energy of Node}

Now, we equate the presentation of our suggested procedure with other Internet of Things protocols like Optimized Ad-hoc On-demand Multipath Distance Vector with Internet of things (AOMDV-IOT) [16], and energy-efficient probabilistic routing (EEPR) [17]. In each approach, we take into account the nodes residual energy and also the variation in energy. Figure 4, depicts the residual energy of other protocols, and we find that the in all of these with increasing figure of sensors the residual energy decreases. Yet we observe that suggested procedure (EEN) has supreme residual energy in comparison to other algorithms.

Table 2: Energy consumption of EEN Protocol

\begin{tabular}{|c|c|c|c|}
\hline Transmission & \multicolumn{3}{|c|}{ Energy consumption(in Joules) } \\
\cline { 2 - 4 } $\begin{array}{c}\text { Rounds (in } \\
\text { No) }\end{array}$ & Shortest & Average & Longest \\
\hline 0 & & & \\
\hline 4 & 0 & 0 & 0 \\
\hline 8 & 7 & 13 & 20 \\
\hline 12 & 12 & 20 & 30 \\
\hline 16 & 18 & 25 & 34 \\
\hline 20 & 20 & 28 & 37 \\
\hline 24 & 22 & 30 & 40 \\
\hline 28 & 22 & 32 & 42 \\
\hline 32 & 20 & 33 & 44 \\
\hline
\end{tabular}

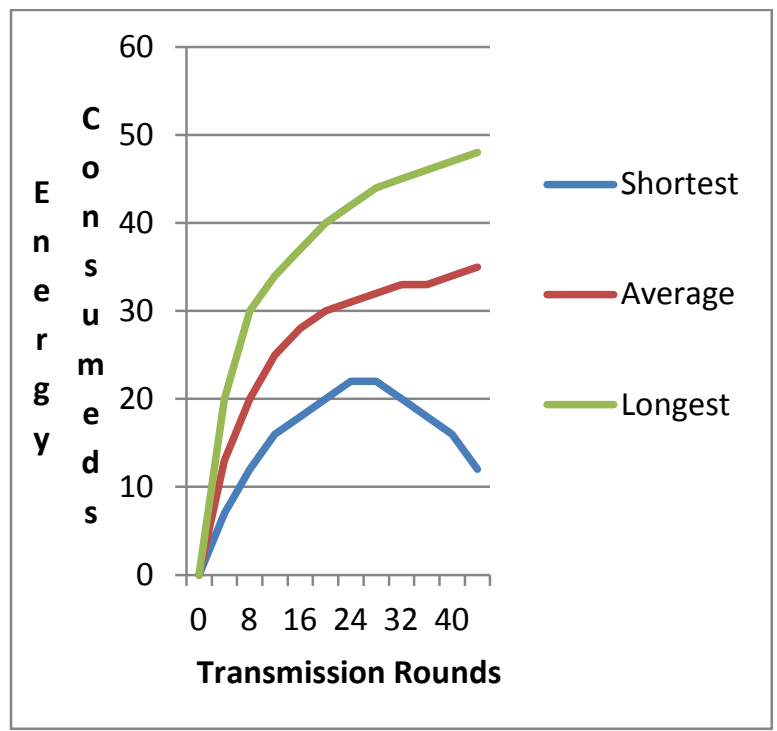

Figure 3: Path determination using EEN Algorithm

Table 3: Comparison between EEN and other IOT protocols

\begin{tabular}{|c|c|c|c|}
\hline \multirow{2}{*}{$\begin{array}{c}\text { No. of } \\
\text { sensor } \\
\text { nodes }\end{array}$} & \multicolumn{3}{|c|}{ Residual energy of nodes } \\
\cline { 2 - 4 } & EEN & AOMDV & EERP \\
\hline 0 & 100 & 98 & 96 \\
\hline 40 & 100 & 95.8 & 95 \\
\hline 80 & 100 & 92 & 96.8 \\
\hline 120 & 97.4 & 88 & 94 \\
\hline 160 & 95 & 77.8 & 89.2 \\
\hline 200 & 92.3 & 72 & 82 \\
\hline 240 & 85 & 68 & 70 \\
\hline 280 & 81.2 & 61.3 & 53.5 \\
\hline 320 & 78 & 58 & 50 \\
\hline 360 & 76 & 55 & 44 \\
\hline
\end{tabular}




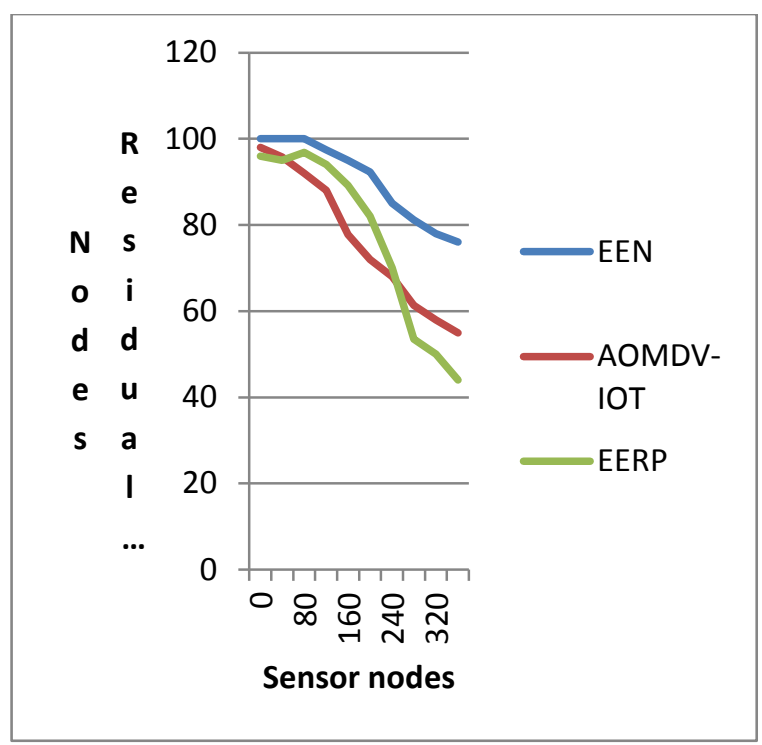

Figure 4: Residual Energy Consumption

After comparing our result with Node Level Energy Efficiency algorithm [18], we can say that our suggested procedure has maximum residual energy in comparison with other approaches.

\section{CONCLUSION}

In this paper, our proposed algorithm has been projected to expand the effectiveness of nodes in Internet of Things. Our approach helps in discovery of the direct path. The route is found out by using residual energy, transmission count and number of node counts. The, path detection procedure is lead using residual energy and counting the nodal paths. The proposed algorithm helps in finding the shortest path when the direction-finding setup postponement is better due to which the direction-finding accomplishment is decreased. Furthermore, EEN algorithm guarantees an improved efficient usage of nodal energies. To define the efficiency of our suggested EEN algorithm, the $\mathrm{C}$ language is used along arduino UN-R3 board and centered on attained data; results are plotted using gnu plot. Depending on the investigational fallouts, we substantiate that our EEN algorithm has lengthier lifespan and devours the residual energy of the nodes much more evenly and gives better performance in comparison to AOMDV-IOT and EEPR in respect of energy effectiveness.

As future work, we will further study the behaviour of the proposed EEN algorithm, investigate more security mechanisms and explore the possibilities of employing it at an even larger scale in order to optimize our system .

\section{REFERENCES}

[1] L. Atzori, A. Iera, and G. Morabito, "The internet of things: a survey," ComputerNetworks, vol. 54,no. 15,pp. 2787-2805, 2010.

[2] ITU Internet Reports, The Internet ofThings, 2005.

[3] O.Vermesan, Internet of Things Strategic Research Roadmap, IERC- European,2011.

[4] G. Lee, "A cluster-based energy-efficient routing protocol without location information for sensor networks," International Journal of Information Processing Systems, vol.1,pp 49-54,2005.
[5] T. Dubey, "Self-localized packet forwarding in wireless sensor networks," International Journal of Information Processing Systems, vol. 9, no. 3, 2013.

[6] A. Sinha, "Performance evaluation of data aggregation for cluster-based wireless sensor network," HumanCentric Computing and Information Sciences, vol. 3, article 13, 2013.

[7] M. Yoon, "An energy-efficient routing protocol using message success rate in wireless sensor networks," KITCS/FTRA Journal Of Convergence, vol. 4, no. 1, 2013

[8] M.U. Farooq, Muhammad Waseem, Sadia Mazhar , Anjum Khairi , Talha Kamal (2015) "A Review on Internet of Things (IoT"). International Journal of Computer Applications (0975 8887) Volume 113 - No. 1.

[9] Ortiz AM, Hussein D, Soochang P, Han SN, Crespi N, et al. (2014) The cluster between internet of things and social networks: Review and research challenges. Internet of Things Journal IEEE 1: 206-215.

[10] Abdul Razaque and Khaled M. Elleithy (2014) EnergyEfficient Boarder Node Medium Access Control, ISSN 1424-8220.

[11] Razaque A, Elleithy KM (2014) Energy-efficient boarder node medium access control protocol for wireless sensor networks. Sensors (Basel) 14: 5074-5117.

[12] Komal Singh, Arun Aggarwal,(2016) "Survey on Optimization Techniques of RFID for Internet of Things". International Journal of Computer Applications (0975 - 8887) Volume 148 - No.9, August 2016.

[13] Abdul Razaque and Khaled Elleith (2015) "Nomenclature of Medium Access Control Protocol over Wireless Sensor Networks" http://dx.doi.org/10.1080/02564602.2015.1057769

[14] Sang-Hyun Park, Seungryong Cho and Jung-Ryun Lee (2014) "Energy-Efficient Probabilistic Routing Algorithm for Internet of Things" : Journal of Applied Mathematics Volume 2014, Article ID 213106, 7 pages .http://dx.doi.org/10.1155/2014/213106.

[15] Mayur Mhaske1, Sayali Shelke1,Bhakti Kulkarni1,Ravindra Salunke (2016): "Compressed Sensing for IOT Application": International Journal of Engineering Technology, Management and Applied Sciences, Volume 4, Issue 2, ISSN 2349-4476.

[16] Chetal. S. Patil, Kanaksing. N. Pawar, (2016) "A Review On: Protocols and Standards in Different Application Areas of IOT", IJARCCE Vol. 5, Issue 2, ISSN (Online) 2278-1021.

[17] Park SH, Cho S, Lee JR (2014) Energy-Efficient Probabilistic Routing Algorithm for Internet of Things. Journal of Applied Mathematics. pp: 1-7.

[18] Vellanki M, Kandukuri SPR and Razaque A (2016) Node Level Energy Efficiency Protocol for Internet of Things: Journal of Theoretical \& Computational Science: DOI: 10.4172/2376-130X.1000140. 